\title{
NMR studies of membrane proteins
}

\author{
Rob Kaptein ${ }^{1}$ - Gerhard Wagner ${ }^{2}$
}

Published online: 4 April 2015

(C) Springer Science+Business Media Dordrecht 2015

The functional importance of proteins that interact with biological membranes can hardly be overestimated. About half of the medicinal drug targets are membrane proteins. Nevertheless, the structural biology of these proteins has been very challenging as only a little more than 500 unique membrane protein structures are present in the PDB (out of 100,000 structures) and also accessible in a membrane protein database (http://blanco.biomol.uci.edu/mpstruc/). About $16 \%$ of these have been derived by NMR spectroscopy. While NMR is limited by molecular size it has the unparalleled capability of observing internal mobility, which can be analyzed at atomic resolution once resonance assignments are obtained. The main obstacles for assignment, dynamics studies and structure determination are low overexpression levels and a high content of hydrophobic amino acids, necessary for embedding into a biological membrane. Thus, some membrane mimetic must always be part of the protein preparation, both for NMR and for X-ray crystallography. The right choice of the membrane mimetic is crucial and is clearly more difficult than finding optimal conditions for studies of soluble proteins. There are numerous options of conditions to select from, such as kind and mixture of detergents and lipids, their concentration, and the relative protein-to-surfactant ratio.

Initial NMR studies of integral membrane proteins focused primarily on $\beta$-barrel folds, which can be characterized relatively well by backbone contacts (Arora et al.

Gerhard Wagner

gerhard_wagner@hms.harvard.edu

1 Bijvoet Centre, Utrecht University, 3584 CH Utrecht, The Netherlands

2 Department of Biological Chemistry, Harvard Medical School, Boston, MA 02115, USA
2001; Fernandez et al. 2001; Hwang et al. 2002). It required more effort to determine structures of helical polytopic membrane proteins where strand topologies have to be established through side-chain contacts (Zhou et al. 2008). In recent years, however, we have witnessed major advances in NMR methodology, which allowed more rapid assignment and structure determination of even helical membrane proteins up to the range of $30-40 \mathrm{kDa}$. For solution NMR an extensive isotope labeling scheme enabled (Nietlispach and Gautier 2011) to determine a 7 TM helical receptor protein in a micellar environment. Addressing the concern that detergents may have a deleterious effect on membrane proteins, in particular for the extramembrane moieties or interacting soluble proteins, phospholipid nanodiscs have been used as a membrane mimetic, which extended the scope of solution NMR (Gluck et al. 2009; Raschle et al. 2009; Shenkarev et al. 2009). Nanodiscs are patches of phospholipid bilayers surrounded by dimeric fragments of a membrane scaffolding protein (Msp) derived from apolipoprotein A1 (Bayburt et al. 1998; Bayburt et al. 2002). Nanodiscs can better stabilize membrane proteins compared to micelles or bicelles (Etzkorn et al. 2013). However, the use of nanodiscs is no magic bullet and also requires extensive optimization of sample conditions as is shown in several articles of this Special Issue. Moreover, the Msp together with the phospholipids add to the total molecular weight, which limits the use of nanodiscs for solution NMR. For solid-state (ss) NMR, there is no principal size limit, which allows a large choice of membrane preparations to be used. In practice, resonance crowding also presents a size limitation for ssNMR unless overcome with creative labeling schemes. Traditionally, ssNMR studies involved orientation of a protein-containing bilayer membrane between glass plates (Gong et al. 2007; Opella 1997). However, the recent boom 
in protein structural studies using magic angle spinning (MAS) ssNMR (Rienstra et al. 2002) together with deuteration benefitted membrane proteins as well (Reif 2012). This Special Issue provides illustrations of all these methods and combinations thereof.

Opella's group has pioneered structural studies of membrane proteins and peptides using both solution and solid-state NMR (Opella 1997; Gong et al. 2007). Here they describe a method to introduce metal-chelating unnatural amino acids into membrane proteins in order to use the powerful structural constraints provided by paramagnetic relaxation enhancement (PRE) and pseudocontact shifts (PCSs) (Park et al. 2014). For the chemokine receptor CXCR1 solubilized in DHPC micelles the authors apply the method successfully to characterize ligandbinding by CXCR1.

Nietlispach and his coworkers (Crick et al. 2015) have also used lanthanide-binding tags to study an integral membrane protein, the 7 trans-membrane helical microbial receptor pSRII, in micellar solution. Combined with selective methyl group labeling the structure of the protein was determined based on a large number of PCSs, sparse NOE's, and H-bond constraints. This protocol looks very promising for structural studies of even larger membrane proteins.

To obtain more sensitive probes of membrane protein studies Maduke (Abraham et al. 2015) places ${ }^{13} \mathrm{C}$ methyl groups on methionines, lysines and engineered cysteines. Using solution NMR they study the $100 \mathrm{kDa}$ dimer of a chloride channel that catalyzes the exchange of $\mathrm{Cl}^{-}$for $\mathrm{H}^{+}$across cellular membranes. The authors aim to monitor structural and dynamic changes due to $\mathrm{Cl}^{-}$and $\mathrm{H}^{+}$binding, which are essentially invisible in the crystal structure arguably due to the packing in the crystal lattice but would be detectible by solution NMR. Indeed, $\mathrm{H}^{+}$ dependent spectral changes are observed in particular in a region assigned to the inner gate of the $\mathrm{Cl}^{-}$permeation pathway.

The group of Usachev at Kazan Federal University shows that protegrin- 2 forms a two-stranded antiparallel $\beta$ sheet that is dimeric in DPC micelles (Usachev et al. 2014). Their results shed light on the mechanism of pore formation of this anti-microbial peptide.

The paper by Bax and coworkers (Roche et al. 2015) describes the interaction between several HIV-1 gp41 constructs and DPC micelles. In particular the ectodomain of gp41 plays a major role in the membrane fusion process that enables entry of the HIV-1 virus in host cells. Using

${ }^{15} \mathrm{~N}$-relaxation and chemical shift perturbation data the authors now characterize the pre-fusion state of the gp41 ectodomain in the presence of detergent. The work may have important implications for the development of antifusion inhibitors.
Several manuscripts explore the use on phospholipid nanodiscs for studies of integral membrane proteins. Wagner's group has recently introduced small phospholipid nanodiscs to obtain well-resolved liquid state NMR spectra of membrane proteins (Hagn et al. 2013; Raschle et al. 2009). Here they use these nanodiscs to determine a high-resolution structure of the $\beta$-barrel protein OmpX (Hagn and Wagner 2014). Side-chain assignments were obtained from specifically labeled protein samples. Protein structures were calculated from a large array of data including (back-calculated) NOEs, residual dipolar couplings, and PREs. In addition, the precise position and tilt of the protein in the membrane could be established using NOEs to lipids and PREs from $\mathrm{Gd}^{3+}$-labeled lipids.

Different membrane-mimicking environments including nanodiscs have been compared by the group of Tamm (Kucharska et al. 2015). They studied two bacterial outer membrane proteins, OprG and OprH in micelles, bicelles and nanodiscs of five different sizes. They stress the importance of optimizing lipids and the benefit of eliminating flexible loops.

An interesting hybrid method combining solution- and solid-state NMR procedures is described by Marassi and coworkers (Ding et al. 2015). They show that membrane proteins reconstituted in lipid bilayer nanodiscs can be reversibly sedimented for analysis by ssNMR without the need for precipitation or lyophilization. The pellets can be resuspended for solution NMR studies or functional assays. This protocol is illustrated with the outer membrane protein Ail from the pathogen Yersinia pestis. High quality ssNMR spectra were obtained.

A recurring issue in membrane protein structural biology is the role of the membrane mimetic on the structure and function of the bound protein or peptide. The group of Ulrich (Misiewicz et al. 2015) has addressed this issue by studying structure and dynamics of the peptide BP100 as a function of lipid composition. A large range of synthetic phospholipid bilayers was tested as well as membranes from erythrocytes and from the bacterium M. luteus. An ssNMR study using macroscopically oriented membrane preparations showed no major differences in peptide structure and its orientation in the lipid. The observed membrane thinning effect of the peptide may be related to its anti-microbial action.

Voltage-dependent anion channels (VDACs) transport ADP, ATP, and other metabolites across the mitochondrial membrane. Two papers in this Special Issue deal with VDAC isoforms 1 and 2. The structure of VDAC1 has previously been solved by solution NMR and X-ray crystallography (Bayrhuber et al. 2008; Hiller et al. 2008; Ujwal et al. 2008). The group of Griffin (Eddy et al. 2015) has now studied VDAC1 by MAS ssNMR. The protein reconstituted in DMPC lipid bilayers forms 2D crystals 
yielding highly resolved NMR spectra. The partial assignment of 90 residues (out of 273) allowed already a secondary structure comparison with earlier data and is a first step towards a full structural characterization.

Lange and coworkers (Gattin et al. 2014) report a MAS ssNMR study of VDAC2. The work is supported by electrophysiology and MD simulations. Structural differences between human VDAC2 and its isoform hVDAC1 are minor, but VDAC2 appears to have a higher plasticity.

The mechanism, by which outer membrane proteins are inserted in Gram-negative bacteria is addressed in two manuscripts. BamA, the major component of the barrel assembly machine Bam consists of a 16-stranded transmembrane $\beta$-barrel and 5 periplasmic PORTA domains. Although there is crystal structure information available, there are many open questions and controversies. Thus, Baldus reports solution and solid-state NMR experiments of fragments of BamA transmembrane and periplasmic domains to resolve these questions (Sinnige et al. 2014). Hiller describes procedures for incorporating full BamA in micelles, bicelles and nanodiscs and reports assignments for parts of the protein (Morgado et al. 2015). The preparation schemes provide the groundwork for further structural and functional studies of membrane proteins.

The mobility of a G-protein coupled receptor in a native-like model membrane is studied by Huster (Thomas et al. 2015) using solid-state NMR. Analyzing order parameters the authors find relatively high rotational mobility primarily around an axis perpendicular to the membrane. The receptor is slightly more rigid in mono-unsaturated than in saturate phospholipids, which the authors relate to the shorter chain lengths.

Dynamic nuclear polarization (DNP) has caused much excitement considering the potential of overcoming the problem of NMR's low sensitivity. To exploit this technology for membrane proteins McDermott and coworkers have covalently attached a pair of spin labels to the interaction interface of gramicidin A (Wylie et al. 2015). Using DNP the authors observe a sixfold signal enhancement. Besides signal enhancement this approach is suitable for studies of interactions within oligomers.

Chou and coworkers (Dev et al. 2015) analyze the mechanism by which the $\mathrm{p} 7$ channel of Hepatitis $\mathrm{C}$ virus promotes cation permeation. Using hydrogen exchange and relaxation dispersion methods the authors show that the lumen of the funnel-like channel is fully water accessible. Furthermore, residues near the asparagine ring at the narrow end of the channel exhibit large chemical exchangetype characteristics. This supports that the funnel end is highly dynamic. This study demonstrates the unique capability of NMR to characterize membrane-protein mobility and is expected to find many followers.
In conclusion, several novel results and methodological advances in membrane protein NMR are reported in this Special Issue. Overall, it provides an excellent representation of the current state-of-the-art of this field. Full de novo structure determination of integral membrane proteins in the range $30-50 \mathrm{kDa}$ and studies of their dynamical behavior are now clearly feasible, both by solution and solid-state NMR. In addition, detailed mechanistic questions on the function of much larger membrane proteins can be addressed, in particular by ssNMR methods.

\section{References}

Abraham SJ et al (2015) ${ }^{13} \mathrm{C}$ NMR detects conformational change in the $100-\mathrm{kD}$ membrane transporter ClC-ec1. J Biomol NMR. doi: 10.1007/s10858-015-9898-7

Arora A, Abildgaard F, Bushweller JH, Tamm LK (2001) Structure of outer membrane protein A transmembrane domain by NMR spectroscopy. Nat Struct Biol 8:334-338

Bayburt TH, Carlson JW, Sligar SG (1998) Reconstitution and imaging of a membrane protein in a nanometer-size phospholipid bilayer. J Struct Biol 123:37-44

Bayburt TH, Grinkova YV, Sligar SG (2002) Self-assembly of discoidal phospholipid bilayer nanoparticles with membrane scaffold proteins. Nano Lett 2:853-856

Bayrhuber M et al (2008) Structure of the human voltage-dependent anion channel. Proc Natl Acad Sci USA 105:15370-15375

Crick DJ, Wang JX, Graham B, Swarbrick JD, Mott HR, Nietlispach D (2015) Integral membrane protein structure determination using pseudocontact shifts. J Biomol NMR. doi:10.1007/s10858015-9899-6

Dev J, Bruschweiler S, Ouyang B, Chou JJ (2015) Transverse relaxation dispersion of the $\mathrm{p} 7$ membrane channel from hepatitis $\mathrm{C}$ virus reveals conformational breathing. J Biomol NMR. doi:10.1007/s10858-015-9912-0

Ding Y, Fujimoto LM, Yao Y, Marassi FM (2015) Solid-state NMR of the Yersinia pestis outer membrane protein Ail in lipid bilayer nanodiscs sedimented by ultracentrifugation. J Biomol NMR. doi:10.1007/s10858-014-9893-4

Eddy MT et al (2015) Lipid bilayer-bound conformation of an integral membrane beta barrel protein by multidimensional MAS NMR. J Biomol NMR. doi:10.1007/s10858-015-9903-1

Etzkorn M, Raschle T, Hagn F, Gelev V, Rice AJ, Walz T, Wagner G (2013) Cell-free expressed bacteriorhodopsin in different soluble membrane mimetics: biophysical properties and NMR accessibility. Structure 21:394-401

Fernandez C, Hilty C, Bonjour S, Adeishvili K, Pervushin K, Wuthrich K (2001) Solution NMR studies of the integral membrane proteins OmpX and OmpA from Escherichia coli. FEBS Lett 504:173-178

Gattin Z et al (2014) Solid-state NMR, electrophysiology and molecular dynamics characterization of human VDAC2. J Biomol NMR. doi:10.1007/s10858-014-9876-5

Gluck JM, Wittlich M, Feuerstein S, Hoffmann S, Willbold D, Koenig BW (2009) Integral membrane proteins in nanodiscs can be studied by solution NMR spectroscopy. J Am Chem Soc 131:12060-12061

Gong XM, Franzin CM, Thai K, Yu J, Marassi FM (2007) Nuclear magnetic resonance structural studies of membrane proteins in micelles and bilayers. Methods Mol Biol 400:515-529 
Hagn F, Wagner G (2014) Structure refinement and membrane positioning of selectively labeled $\mathrm{OmpX}$ in phospholipid nanodiscs. J Biomol NMR. doi:10.1007/s10858-014-9883-6

Hagn F, Etzkorn M, Raschle T, Wagner G (2013) Optimized phospholipid bilayer nanodiscs facilitate high-resolution structure determination of membrane proteins. J Am Chem Soc 135:1919-1925

Hiller S, Garces RG, Malia TJ, Orekhov VY, Colombini M, Wagner $G$ (2008) Solution structure of the integral human membrane protein VDAC-1 in detergent micelles. Science 321:1206-1210

Hwang PM et al (2002) Solution structure and dynamics of the outer membrane enzyme PagP by NMR. Proc Natl Acad Sci USA 99:13560-13565

Kucharska I, Edrington TC, Liang B, Tamm LK (2015) Optimizing nanodiscs and bicelles for solution NMR studies of two b-barrel membrane proteins. J Biomol NMR. doi:10.1007/s10858-0159905-Z

Misiewicz J, Afonin S, Grage SL, van den Berg J, Strandberg E, Wadhwani P, Ulrich AS (2015) Action of the multifunctional peptide BP100 on native biomembranes examined by solid-state NMR. J Biomol NMR. doi:10.1007/s10858-015-9897-8

Morgado L, Zeth K, Burmann BM, Maier T, Hiller S (2015) Characterization of the insertase BamA in three different membrane mimetics by solution NMR spectroscopy. J Biomol NMR. doi:10.1007/s10858-015-9906-y

Nietlispach D, Gautier A (2011) Solution NMR studies of polytopic alpha-helical membrane proteins. Curr Opin Struct Biol 21:497-508

Opella SJ (1997) NMR and membrane proteins. Nat Struct Biol 4(Suppl):845-848

Park SH, Wang VS, Radoicic J, De Angelis AA, Berkamp S, Opella SJ (2014) Paramagnetic relaxation enhancement of membrane proteins by incorporation of the metal-chelating unnatural amino acid 2-amino-3-(8-hydroxyquinolin-3-yl)propanoic acid (HQA). J Biomol NMR. doi:10.1007/s10858-014-9884-5

Raschle T, Hiller S, Yu TY, Rice AJ, Walz T, Wagner G (2009) Structural and functional characterization of the integral membrane protein VDAC-1 in lipid bilayer nanodiscs. J Am Chem Soc 131:17777-17779
Reif B (2012) Ultra-high resolution in MAS solid-state NMR of perdeuterated proteins: implications for structure and dynamics. J Magn Reson 216:1-12

Rienstra CM et al (2002) De novo determination of peptide structure with solid-state magic-angle spinning NMR spectroscopy. Proc Natl Acad Sci USA 99:10260-10265

Roche J, Louis JM, Aniana A, Ghirlando R, Bax A (2015) Complete dissociation of the HIV-1 gp41 ectodomain and membrane proximal regions upon phospholipid binding. J Biomol NMR. doi:10.1007/s10858-015-9900-4

Shenkarev ZO et al (2009) Lipid-protein nanodiscs: possible application in high-resolution NMR investigations of membrane proteins and membrane-active peptides. Biochemistry (Mosc) 74:756-765

Sinnige T, Weingarth M, Renault M, Baker L, Tommassen J, Baldus M (2014) Solid-state NMR studies of full-length BamA in lipid bilayers suggest limited overall POTRA mobility. J Mol Biol 426:2009-2021

Thomas L, Kahr J, Schmidt P, Krug U, Scheidt HA, Huster D (2015) The dynamics of the $G$ protein-coupled neuropeptide $Y 2$ receptor in monounsaturated membranes investigated by solidstate NMR spectroscopy. J Biomol NMR. doi:10.1007/s10858014-9892-5

Ujwal R et al (2008) The crystal structure of mouse VDAC1 at $2.3 \mathrm{~A}$ resolution reveals mechanistic insights into metabolite gating. Proc Natl Acad Sci USA 105:17742-17747

Usachev KS, Efimov SV, Kolosova OA, Filippov AV, Klochkov VV (2014) High-resolution NMR structure of the antimicrobial peptide protegrin-2 in the presence of DPC micelles. J Biomol NMR. doi:10.1007/s10858-014-9885-4

Wylie BJ, Dzikowski BG, Pawsey S, Caporini M, Rosey M, Freed JH, McDemott A (2015) Dynamic nuclear polarization of membrane proteins: covalently bound spi-labels at protein-protein interfaces. J Biomol NMR. doi:10.1007/s10858-015-9919-6

Zhou Y et al (2008) NMR solution structure of the integral membrane enzyme DsbB: functional insights into DsbB-catalyzed disulfide bond formation. Mol Cell 31:896-908 